\title{
THE EXISTENGE OF A DISTRIBUTION FUNCTION FOR AN ERROR TERM RELATED TO THE EULER FUNCTION
}

\author{
PAUL ERDÖS AND H. N. SHAPIRO
}

1. Introduction. The average order of the Euler function $\phi(n)$, the number of integers less than $n$ which are relatively prime to $n$, raises many difficult and still unanswered questions. Thus, for

$$
R(x)=\sum_{n \leqslant x} \phi(n)-\frac{3}{\pi^{2}} x^{2}
$$

and

$$
H(x)=\sum_{n \leqslant x} \frac{\phi(n)}{n}-\frac{6}{\pi^{2}} x,
$$

it is known that $R(x)=O(x \log x)$ and $H(x)=O(\log x)$. However, though these results are quite old, they were not improved until recently. Walfisz (1) has given the outline of a proof of

$$
R(x)=O\left(x(\log x)^{3 / 4}(\log \log x)^{2}\right) .
$$

On the other hand it is known (3) that

$$
R(x) \neq O(x \log \log \log x) .
$$

In this direction it was proved in (4) that each of the following inequalities holds for infinitely many integral $x$ ( $c$ a certain positive constant):

$$
\begin{aligned}
& R(x)>c x \log \log \log \log x, \\
& R(x)<-c x \log \log \log \log x, \\
& H(x)>c \log \log \log \log x, \\
& H(x)<-c \log \log \log \log x .
\end{aligned}
$$

In this paper we propose to continue the study of the error function $H(x)$, and will prove that $H(x)$ possesses a continuous distribution function. By this we mean that for $N(n, u)=$ the number of $m \leqslant n$ such that $H(m) \geqslant u$, we have for each $u,-\infty<u<\infty$, that the limit

$$
\lim _{n \rightarrow \infty} \frac{N(n, u)}{n}=F(u)
$$

exists; and the non-increasing function $F(u)$ is continuous for all $u$.

In the case of additive arithmetic functions, necessary and sufficient conditions for the existence of a distribution function are known $(\mathbf{5} ; \mathbf{6})$. The methods used in (5) to establish the sufficient conditions seem to apply in a fairly general way for establishing the existence of a continuous distribu-

Received May 19, 1954. 
tion function even for a function which is not additive (7). This method serves also as the basic framework of the proof given here for the existence of a continuous distribution function for $H(x)$.

There are essentially three steps. First, we introduce for each integer $k \geqslant 1$, the function

$$
H_{k}(x)=\sum_{n \leqslant x} \frac{\phi\left(\left(n, A_{k}\right)\right)}{\left(n, A_{k}\right)}-x \prod_{p \leqslant p_{k}}\left(1-\frac{1}{p^{2}}\right),
$$

where

$$
A_{k}=\prod_{p \leqslant p_{k}} p
$$

where $p_{k}$ is the $k$ th prime. It is then shown that for each $u$, with fixed $k$, if $N_{k}(n, u)$ is the number of $m \leqslant n$ such that $H_{k}(x) \geqslant u$, the limit

$$
\lim _{n \rightarrow \infty} \frac{N_{k}(n, u)}{n}=F_{k}(u)
$$

exists. We then see that (1.9) follows if we can show that, for a given $u$ and any $\epsilon>0$, the inequality

$$
\left|N(n, u)-N_{k}(n, u)\right|<\epsilon n
$$

holds for each $k \geqslant k_{0}=k_{0}(\epsilon)$ and all $n \geqslant n_{0}=n_{0}(k)$. For from (1.12) we have

$$
\left|\sup \frac{N(n, u)}{n}-\lim _{n \rightarrow \infty} \frac{N_{k}(n, u)}{n}\right| \leqslant \epsilon, \quad\left|\inf \frac{N(n, u)}{n}-\lim _{n \rightarrow \infty} \frac{N_{k}(n, u)}{n}\right| \leqslant \epsilon,
$$

for $k \geqslant k_{0}$. This in turn gives

$$
\left|\sup \frac{N(n, u)}{n}-\inf \frac{N(n, u)}{n}\right| \leqslant 2 \epsilon,
$$

and the existence of the limit (1.9) follows.

The next two steps of the proof are devoted to establishing (1.12). This asserts that the number of $m \leqslant n$ such that either

or

$$
\text { ( } \alpha) \quad H(m)<u \text { and } H_{k}(m) \geqslant u
$$

$$
\text { (B) } H(m) \geqslant u \text { and } H_{k}(m)<u
$$

is less than $\epsilon n$ for each $k \geqslant k_{0}$, and sufficiently large $n$. It suffices (since the argument is the same for the other case) to consider only the case $(\alpha)$. At this point the second step of the proof comes in. It is proved that, given any $\delta>0$, $\epsilon>0$, for $k$ fixed sufficiently large, and $n$ sufficiently large,

$$
\left|H(m)-H_{k}(m)\right|<\delta
$$

except for at most $\frac{1}{2} \epsilon n$ integers $m \leqslant n$. Thus in case $(\alpha)$,

$$
H(m)<u-\delta, \quad H_{k}(m) \geqslant u
$$

can hold for at most $\frac{1}{2} \epsilon n$ integers $m \leqslant n$. Hence we need consider only those $m$ for which

$$
u-\delta<H(m)<u .
$$


This then brings us to the third step of the proof. It is shown that given $\epsilon>0$ there exists a $\delta>0(\delta=\delta(\epsilon)$, independent of $u)$, such that for sufficiently large $n$, the number of $m \leqslant n$ such that (1.14) holds is less than $\frac{1}{2} \epsilon n$. This clearly completes the proof of the existence of $F(u)$. Furthermore, the result of this third step implies that for a fixed $u$, given any $\epsilon>0$, there is a $\delta=\delta(\epsilon)$ such that $0 \leqslant F(u-\delta)-F(u)<\epsilon$, which yields the continuity of $F(u)$.

The main component of the argument used to carry out this last step is the result that, for any fixed integer $l$, the function

$$
\Phi_{l}(x)=\frac{\phi(x)}{x}+\frac{\phi(x+1)}{x+1}+\ldots+\frac{\phi(x+l)}{x+l}
$$

has a continuous distribution function. Though we shall not bother to delineate the proof of this, it is contained in the arguments given. The idea in the proof of the result desired in the third step is that its negation would for some $l$ imply the existence of a discontinuity in the distribution function of $\Phi_{l}(x)$.

2. First step: The existence of $F_{k}(u)$. We have

$$
\begin{aligned}
\sum_{n \leqslant x} \frac{\phi\left(\left(n, A_{k}\right)\right)}{\left(n, A_{k}\right)} & =\sum_{n \leqslant x} \sum_{d \mid\left(n, A_{k}\right)} \frac{\mu(d)}{d} \\
& =\sum_{d \mid A_{k}} \frac{\mu(d)}{d}\left[\frac{x}{d}\right] \\
& =x \sum_{d \mid A_{k}} \frac{\mu(d)}{d^{2}}-\sum_{d \mid A_{k}} \frac{\mu(d)}{d}\left\{\frac{x}{d}\right\},
\end{aligned}
$$

where $\{z\}=z-[z]$ denotes the fractional part of $z$. This in turn yields, from (1.10),

$$
H_{k}(x)=-\sum_{d \mid A_{k}} \frac{\mu(d)}{d}\left\{\frac{x}{d}\right\}
$$

Since $\{x / d\}$ is, for fixed $d$, a periodic function of $x$ with period $d$, we see from (2.1) that $H_{k}(x)$ is a periodic function of $x$ with $A_{k}$ as a period. Thus we have

so that

$$
N_{k}(n, u)=\sum_{\substack{m \leqslant n \\ H_{k}(m) \geqslant u}} 1=\frac{n}{A_{k}} \sum_{\substack{m \leqslant A_{k} \\ H_{k}(m) \geqslant u}} 1+O(1),
$$

$$
\lim _{\rightarrow \infty} \frac{N_{k}(n, u)}{n}=\frac{1}{A_{k}} N_{k}\left(A_{k}, u\right)=F_{k}(u)
$$

exists.

3. Second step. We will prove in this section that, given any $\eta>0$, for each $k \geqslant k_{0}=k_{0}(\eta)$, and all $x \geqslant x_{0}=x_{0}(k)$, we have

$$
\sum_{n=1}^{x}\left(H(n)-H_{k}(n)\right)^{2}<\eta x
$$


From this it follows that if $M(x)$ is the number of $n \leqslant x$ such that

$$
\left|H(n)-H_{k}(n)\right|>\delta,
$$

$M(x)<\eta x / \delta^{2}$, which yields the statement concerning (1.13).

(3.1) is established in a rather straightforward fashion in the following sequence of lemmas.

LEMMA 3.1.

$$
\sum_{n \leqslant x} H^{2}(n) \sim\left(\frac{1}{2 \pi^{2}}+\frac{6}{\pi^{4}}\right) x .
$$

Proof. This is essentially Lemma 12 of (8), which asserts that

$$
\int_{1}^{x} H^{2}(u) d u \sim \frac{x}{2 \pi^{2}} .
$$

The passage from (3.3) to (3.2) is simple and we omit it. In passing it is perhaps of some interest to note that (3.3) is proved by means of a method of Walfisz (2), and seems to be slightly "deeper" than the rest of our estimates which require only elementary methods together with a strong form of the prime number theorem.

LEMMA 3.2 .

$$
\sum_{n \leqslant x} H_{k}^{2}(n) \sim \alpha_{k} x
$$

where

$$
\begin{aligned}
\alpha_{k}= & \frac{1}{12} \sum_{\substack{d_{1}\left|A_{k} \\
d_{2}\right| A_{k}}} \frac{\mu\left(d_{1}\right) \mu\left(d_{2}\right)}{d_{1}^{2} d_{2}^{2}}\left(d_{1}, d_{2}\right)^{2} \\
& +\frac{1}{6} \prod_{p \leqslant p_{k}}\left(1-\frac{1}{p^{2}}\right)^{2}+\frac{1}{4} \prod_{p \leqslant p_{k}}\left(1-\frac{1}{p}\right)^{2} \\
& -\frac{1}{2} \prod_{p \leqslant p_{k}}\left(1-\frac{1}{p}\right)\left(1-\frac{1}{p^{2}}\right) .
\end{aligned}
$$

Furthermore,

$$
\lim _{k \rightarrow \infty} \alpha_{k}=\frac{1}{2 \pi^{2}}+\frac{6}{\pi^{4}} .
$$

Proof. From (2.1) we have

$$
\begin{aligned}
\sum_{n \leqslant x} H_{k}^{2}(n) & =\sum_{n \leqslant x} \sum_{\substack{d_{1}\left|A_{k} \\
d_{2}\right| A_{k}}} \frac{\mu\left(d_{1}\right)}{d_{1}} \frac{\mu\left(d_{2}\right)}{d_{2}}\left\{\frac{n}{d_{1}}\right\}\left\{\frac{n}{d_{2}}\right\} \\
& =\sum_{\substack{d_{1}\left|A_{k} \\
d_{2}\right| A_{k}}} \frac{\mu\left(d_{1}\right) \mu\left(d_{2}\right)}{d_{1} d_{2}} \sum_{n \leqslant x}\left\{\frac{n}{d_{1}}\right\}\left\{\frac{n}{d_{2}}\right\} .
\end{aligned}
$$

Also, 


$$
\begin{aligned}
\sum_{n \leqslant x}\left\{\frac{n}{d_{1}}\right\}\left\{\frac{n}{d_{2}}\right\} & =\sum_{\substack{1 \leqslant i \leqslant d_{1}-1 \\
1 \leqslant j \leqslant d_{2}-1 \\
\left(d_{1}, d_{2}\right) \mid(i-j)}} \frac{\eta j}{d_{1} d_{2}} \sum_{\substack{n \leqslant x \\
n \equiv i\left(d_{1}\right) \\
n \equiv j\left(d_{2}\right)}} 1 \\
& =\frac{1}{d_{1} d_{2}} \sum_{\substack{1 \leqslant i \leqslant d_{1}-1 \\
1 \leqslant j \leqslant d_{2}-1 \\
\left(d_{1}, d_{2}\right) \mid i-j}} i j\left(\frac{x}{\left\{d_{1}, d_{2}\right\}}+O(1)\right) \\
& =\frac{\lambda}{d_{1}^{2} d_{2}^{2}} x \sum_{\substack{1 \leqslant i \leqslant d_{1}-1 \\
1 \leqslant j \leqslant d_{2}-1 \\
\lambda \mid(i-j)}} i j+O(1),
\end{aligned}
$$

where $\lambda=\left(d_{1}, d_{2}\right)$ is greatest common divisor of $d_{1}, d_{2}$, and $\left\{d_{1}, d_{2}\right\}$ is the least common multiple of $d_{1}, d_{2}$.

A simple calculation gives that

$$
\begin{aligned}
\sum_{\substack{1 \leq i \leq d_{1}-1 \\
1 \leq j \leq d_{2}-1 \\
\lambda \backslash i-j}} i j & =\sum_{l=0}^{\lambda-1}\left(\sum_{\substack{i=1 \\
i \equiv l(\lambda)}}^{d_{1}-1} i\right)\left(\sum_{\substack{j=1 \\
j \equiv l(\lambda)}}^{d_{2}-1} j\right) \\
& =\frac{d_{1} d_{2}}{\lambda}\left(\frac{\lambda^{2}}{12}+\frac{1}{6}+\frac{d_{1} d_{2}}{4}-\frac{\left(d_{1}+d_{2}\right)}{4}\right) .
\end{aligned}
$$

Combining (3.7), (3.8) and (3.9) yields

$$
\begin{aligned}
\sum_{n \leqslant x} H_{k}^{2}(n)= & x \sum_{\substack{d_{1}\left|A_{k} \\
d_{2}\right| A_{k}}} \frac{\mu\left(d_{1}\right)}{d_{1}^{2}} \frac{\mu\left(d_{2}\right)}{d_{2}^{2}}\left\{\frac{\lambda^{2}}{12}+\frac{1}{6}+\frac{d_{1} d_{2}}{4}-\frac{d_{1}}{2}\right\}+O(1) \\
\sim & x\left(\frac{1}{12} \sum_{\substack{d_{1}\left|A_{k} \\
d_{2}\right| A_{k}}} \frac{\mu\left(d_{1}\right)}{d_{1}^{2}} \frac{\mu\left(d_{2}\right)}{d_{2}^{2}}\left(d_{1}, d_{2}\right)^{2}\right. \\
& +\frac{1}{6} \prod_{p \leqslant p_{k}}\left(1-\frac{1}{p^{2}}\right)^{2}+\frac{1}{4} \prod_{p \leqslant p_{k}}\left(1-\frac{1}{p}\right)^{2} \\
& \left.-\frac{1}{2} \prod_{p \leqslant p_{k}}\left(1-\frac{1}{p}\right)\left(1-\frac{1}{p^{2}}\right)\right)
\end{aligned}
$$

which is precisely (3.4) and (3.5). Since

$$
\prod_{p \leqslant p_{k}}\left(1-\frac{1}{p}\right) \rightarrow 0 \text { as } k \rightarrow \infty ; \text { and } \prod_{p}\left(1-\frac{1}{p^{2}}\right)=\frac{6}{\pi^{2}},
$$

it follows from (3.5) that

$$
\lim _{k \rightarrow \infty} \alpha_{k}=\frac{1}{12} \sum_{d_{1}, d_{2}} \frac{\mu\left(d_{1}\right) \mu\left(d_{2}\right)\left(d_{1}, d_{2}\right)^{2}}{d_{1}^{2} d_{2}^{2}}+\frac{6}{\pi^{4}} .
$$

(3.6) then follows from

$$
\sum_{d_{1}, d_{2}} \frac{\mu\left(d_{1}\right) \mu\left(d_{2}\right)\left(d_{1}, d_{2}\right)^{2}}{d_{1}^{2} d_{2}^{2}}=\frac{6}{\pi^{2}}
$$

LEMMA 3.3.

$$
\sum_{n \leqslant x} H(n) H_{k}(n) \sim \beta_{k} x
$$


where

$$
\begin{aligned}
\beta_{k}= & \frac{1}{12} \sum_{\substack{d_{1}\left|A_{k} \\
d_{2}\right| A_{k}}} \frac{\mu\left(d_{1}\right)}{d_{1}^{2}} \frac{\mu\left(d_{2}\right)}{d_{2}^{2}}\left(d_{1}, d_{2}\right)^{2} \\
& -\frac{3}{2 \pi^{2}} \prod_{p \leqslant p_{k}}\left(1-\frac{1}{p}\right)+\frac{1}{\pi^{2}} \prod_{p \leqslant p_{k}}\left(1-\frac{1}{p^{2}}\right) .
\end{aligned}
$$

Furthermore,

$$
\lim _{k \rightarrow \infty} \beta_{k}=\frac{1}{2 \pi^{2}}+\frac{6}{\pi^{4}}
$$

Proof. Setting

$$
M(u)=\sum_{d \leqslant u} \frac{\mu(d)}{d},
$$

since, by the prime number theorem, $M(u)=O\left(\log ^{-c} u\right)$ for any fixed $c>0$, we have for $u v=x$

$$
\begin{aligned}
\sum_{n \leqslant x} \frac{\phi(n)}{n} & =\sum_{d d^{\prime} \leqslant x} \frac{\mu(d)}{d} \\
& =\sum_{d \leqslant u} \frac{\mu(d)}{d}\left[\frac{x}{d}\right]+\sum_{d^{\prime} \leqslant v} M\left(\frac{x}{d^{\prime}}\right)-M(u)[v] \\
& =\frac{6}{\pi^{2}} x-\sum_{d \leqslant u} \frac{\mu(d)}{d}\left\{\frac{x}{d}\right\}+O\left(v \log ^{-2 c} u\right) .
\end{aligned}
$$

Taking $u=x \log ^{-c} x$, we get

$$
H(x)=-\sum_{d \leqslant x \log ^{-} x} \frac{\mu(d)}{d}\left\{\frac{x}{d}\right\}+O\left(\log ^{-c} x\right) .
$$

(This is essentially Lemma 2 of (8).)

From (2.1) and (3.13) we obtain

$$
\sum_{n \leqslant x} H_{k}(n) H(n)=\sum_{\substack{d_{1} \backslash A_{k} \\ d_{2} \leqslant x \log ^{-} \circ x}} \frac{\mu\left(d_{1}\right)}{d_{1}} \frac{\mu\left(d_{2}\right)}{d_{2}} \sum_{n \leqslant x}\left\{\frac{n}{d_{1}}\right\}\left\{\frac{n}{d_{2}}\right\}+O\left(\log ^{-c} x\right) .
$$

Using a slight modification of (3.8) and (3.9) we get

$$
\begin{aligned}
& \sum_{n \leqslant x} H_{k^{\prime}}(n) H(n)= x \sum_{\substack{d_{1} \mid A_{k} \\
d_{2} \leqslant x \log ^{-c_{x}}}} \frac{\mu\left(d_{1}\right)}{d_{1}^{2}} \frac{\mu\left(d_{2}\right)}{d_{2}^{2}}\left\{\frac{\lambda^{2}}{12}+\frac{1}{6}+\frac{d_{1} d_{2}}{4}-\frac{\left(d_{1}+d_{2}\right)}{4}\right\} \\
&+O\left(x \log ^{-c} x\right) \\
& \sim x\left(\frac{1}{12} \sum_{\substack{d_{1}\left|A_{k} \\
d_{3}\right| A_{k}}} \frac{\mu\left(d_{1}\right)}{d_{1}^{2}} \frac{\mu\left(d_{2}\right)}{d_{2}^{2}}\left(d_{1}, d_{2}\right)^{2}\right. \\
&\left.-\frac{3}{2 \pi^{2}} \prod_{p \leqslant p_{k}}\left(1-\frac{1}{p_{k}}\right)+\frac{1}{\pi^{2}} \prod_{p \leqslant p_{k}}\left(1-\frac{1}{p^{2}}\right)\right)
\end{aligned}
$$

which gives (3.10) and (3.11). From this it follows easily that

$$
\lim _{k \rightarrow \infty} \beta_{k}=\frac{1}{2 \pi^{2}}+\frac{6}{\pi^{4}} \text {. }
$$


Applying (3.2), (3.4), and (3.10) we have

$$
\begin{aligned}
\sum_{n \leqslant x}\left(H(n)-H_{k}(n)\right)^{2} & =\sum_{n \leqslant x} H^{2}(n)-2 \sum_{n \leqslant x} H_{k}(n) H(n)+\sum_{n \leqslant x} H_{k}^{2}(n) \\
& \sim x\left\{\frac{1}{2 \pi^{2}}+\frac{6}{\pi^{4}}-2 \beta_{k}+\alpha_{k}\right\} .
\end{aligned}
$$

From (3.6) and (3.12) we see that

$$
\lim _{k \rightarrow \infty}\left(\frac{1}{2 \pi^{2}}+\frac{6}{\pi^{4}}-2 \beta_{k}+\alpha_{k}\right)=0,
$$

and the assertion concerning (3.1) made at the beginning of this section follows immediately.

4. The third step. In this section we propose to prove that, given any $\epsilon>0$, there exists a $\delta>0$ such that the number of $m \leqslant x$ such that

$$
u<H(m)<u+\delta
$$

for some $u$,

is (for sufficiently large $x$ ) less than $\epsilon x$.

We shall suppose that the above statement is false and derive a contradiction. Negating the above assertion yields that for some constant $A>0$ and each $\delta>0$, there exist infinitely many positive integers $x$ (depending possibly on $\delta$ ) such that for some $u$ (depending possibly on $x$ as well as on $u$ ) the number of $m \leqslant x$ such that (4.1) is satisfied is at least $A x$.

Since from (3.2) we have

$$
\sum_{m=1}^{x} H^{2}(m)<c_{1} x
$$

it follows that for these $u=u(x, \delta)$ (we restrict ourselves to $0<\delta<1$ ), we have that either $-2 \leqslant u \leqslant 0$, or from (4.2)

$$
\frac{u^{2}}{4} A x<c_{1} x
$$

so that in any event the possible values of $u=u(x, \delta)$ are bounded. Thus for each $\delta(0<\delta<1)$ we can find an infinite sequence of positive integers $\left\{x_{i}(\delta)\right\}$ such that

$$
\lim _{i \rightarrow \infty} u\left(x_{i}(\delta), \delta\right)=u^{*}(\delta),
$$

where the set of $u^{*}(\delta)$ is also clearly bounded. Thus again we can choose a sequence $\delta_{j} \rightarrow 0$ such that the limit

$$
\lim _{j \rightarrow \infty} u^{*}\left(\delta_{j}\right)=\bar{u}
$$

exists.

Given any $\delta>0$ we can find a $\delta_{j}<\frac{1}{3} \delta$ such that

$$
\left|\bar{u}-u^{*}\left(\delta_{j}\right)\right|<\frac{1}{3} \delta \text {. }
$$


Since from (4.3) we know that, for all sufficiently large $i$,

$$
\left|u^{*}\left(\delta_{j}\right)-u\left(x_{i}\left(\delta_{j}\right), \delta_{j}\right)\right|<\frac{1}{3} \delta,
$$

it follows that for this sequence $\left\{x_{i}\left(\delta_{j}\right)\right\}$ we have

$$
\left|\bar{u}-u\left(x_{i}\left(\delta_{j}\right), \delta_{j}\right)\right|<\frac{2}{3} \delta .
$$

For $m \leqslant x_{i}\left(\delta_{j}\right)$ there are more than $A x_{i}$ integers $m \leqslant x_{i}$ such that

$$
u\left(x_{i}\left(\delta_{j}\right), \delta_{j}\right)<H(m)<u\left(x_{i}\left(\delta_{j}\right), \delta_{j}\right)+\delta_{j} .
$$

But since (4.5) implies that

$$
\bar{u}-\delta<u\left(x_{i}\left(\delta_{j}\right), \delta_{j}\right)<u\left(x_{i}\left(\delta_{j}\right), \delta_{j}\right)+\delta_{j}<\bar{u}+\delta,
$$

it follows that for at least $\frac{1}{2} A x_{i}$ of the $m \leqslant x_{i}$ we have one of the inequalities

$$
\begin{array}{ll}
(\alpha) & \bar{u} \leqslant H(m)<\bar{u}+\delta, \\
(\beta) & \bar{u}-\delta<H(m) \leqslant \bar{u} .
\end{array}
$$

Since at least one of $(\alpha)$ or $(\beta)$ must occur for a sequence of $\delta$ 's approaching 0 , at least one of these is the case for all $\delta>0$. Since the treatment of the other case is exactly the same, we assume $(\alpha)$. Thus we have that, for any $\delta>0$, there exist infinitely many positive integers $n$ such that the number of integers $m \leqslant n$ for which

$$
\bar{u} \leqslant \sum_{r=1}^{m} \frac{\phi(r)}{r}-\frac{6}{\pi^{2}} m<\bar{u}+\delta
$$

is greater than $\frac{1}{2} A n$.

Let $m_{1}<m_{2}<\ldots<m_{t} \leqslant n\left(t>\frac{1}{2} A n\right)$ be the integers $\leqslant n$ which satisfy (4.7). Clearly $m_{i+1}-m_{i}<4 / A$ has at least $\frac{1}{4} A n$ solutions. Thus there exists an integer $l<4 / A$ such that $m_{i+1}-m_{i}=l$ has at least $A^{2} n / 16$ solutions. Furthermore, by extracting a suitable subsequence from our infinite sequence of $n$, we may assume that $l$ is independent of $n$.

The above in turn implies that for any $\delta>0$ there exists an infinite sequence of $n$ such that

$$
\left|\sum_{r=m}^{m+l-1} \frac{\phi(r)}{r}-\frac{6}{\pi^{2}} l\right| \leqslant \delta
$$

has at least $A^{2} n / 16$ solutions $m \leqslant n$. In deriving a contradiction from this, the underlying idea is that this implies that the distribution function (it exists, though we forego a proof of this) of

$$
\frac{\phi(x)}{x}+\ldots+\frac{\phi(x+l-1)}{x+l-1}
$$

would have to have a discontinuity at $6 l / \pi^{2}$, and this in turn would lead to the existence of a discontinuity in the distribution function of $\phi(x) / x$ (which is known to exist and be continuous) (5). 
We set

so that

$$
\begin{gathered}
\frac{\phi_{D}(x)}{x}=\prod_{\substack{p \nmid x \\
p \leqslant D}}\left(1-\frac{1}{p}\right), \\
\mu_{D}(n)= \begin{cases}\mu(n) \text { if } n \text { is divisible only by primes } p \leqslant D, \\
0 \quad \text { otherwise; }\end{cases}
\end{gathered}
$$

and

$$
\frac{\phi_{D}(x)}{x} \geqslant \frac{\phi(x)}{x}
$$

$$
\begin{aligned}
0 & \leqslant \sum_{n \leqslant x}\left\{\frac{\phi_{D}(n)}{n}-\frac{\phi(n)}{n}\right\}=\sum_{d \leqslant x}\left\{\frac{\mu_{D}(d)}{d}-\frac{\mu(d)}{d}\right\}\left[\frac{x}{d}\right] \\
& =x \sum_{d \leqslant x} \frac{\mu_{D}(d)-\mu(d)}{d^{2}}+O(\log x) \\
& \sim x\left(\prod_{p \leqslant D}\left(1-\frac{1}{p^{2}}\right)-\frac{6}{\pi^{2}}\right) .
\end{aligned}
$$

From this it follows that, given $\eta_{1}, \eta_{2}>0$, we can choose $D \geqslant D\left(\eta_{1}, \eta_{2}\right)$ sufficiently large so that for all but $\eta_{1} n$ integers $x \leqslant n$ we have

$$
0 \leqslant \frac{\phi_{D}(x)}{x}-\frac{\phi(x)}{x}<\eta_{2}
$$

Thus, taking $\eta_{2}=\delta / l$ and $\eta_{1}=A^{2} / 32$, we obtain from (4.8) that for each sufficiently large $D$, there exist infinitely many positive integers $n$ such that the inequalities

$$
\left|\sum_{r=m}^{m+l-1} \frac{\phi_{D}(r)}{r}-\frac{6}{\pi^{2}} l\right| \leqslant 2 \delta
$$

and

$$
\left|\frac{\phi(m)}{m}+\sum_{r=m+1}^{m+l-1} \frac{\phi_{D}(r)}{r}-\frac{6}{\pi^{2}} l\right| \leqslant 2 \delta
$$

are satisfied simultaneously by at least $A^{2} n / 32$ integers $m \leqslant n$.

LEMMA 4.1. There exist absolute constants $\rho>0$, and $\delta_{0}>0$ (independent of $D$ ) such that for at least $A^{2} n / 64$ of the solutions $m \leqslant n$ of (4.9) and (4.10) we have for $\delta<\delta_{0}$

$$
\frac{6}{\pi^{2}} l-\sum_{r=m+1}^{m+l-1} \frac{\phi_{D}(r)}{r} \geqslant \rho .
$$

Proof. For if (4.11) is false, (4.10) implies

$$
0 \leqslant \frac{\phi(m)}{m} \leqslant \rho+2 \delta .
$$

Since the distribution function of $\phi(m) / m$ exists and is continuous, for $\rho$ and $\delta$ sufficiently small, (4.12) can have at most $A^{2} n / 64$ solutions $m \leqslant n$. 
Thus we may restrict ourselves to solutions $m$ of (4.9) for which (4.11) holds. Also there is no loss of generality in assuming $\delta<\frac{1}{3} \rho$, as we shall do henceforth.

Next, we discard a certain "small" set of integers. Since

$$
\sum_{m=1}^{n} \sum_{p \mid m} \frac{1}{p}=\sum_{p \leqslant n} \frac{1}{p}\left[\frac{n}{p}\right]<n \sum_{p} \frac{1}{p^{2}}=c_{2} n
$$

it follows that the number of $m \leqslant n$ such that

$$
\sum_{p \mid m+i} \frac{1}{p}<E
$$$$
0 \leqslant i \leqslant l-1 \text {, }
$$

fails to hold is less than $l c_{2} n / E$, which for $E>128 l c_{2} / A^{2}$ is in turn less than $A^{2} n / 128$. Thus for such an $E$ we have an infinite sequence of $n$ such that (4.9), (4.11) and (4.14) hold simultaneously for more than $A^{2} n / 128$ integers $m \leqslant n$.

We now attempt to show that the set of integers $m$ which satisfy (4.9), (4.11) and (4.14) has small density, thereby obtaining a contradiction. For a given integer $m$ define

$$
\lambda(m)=\prod_{\substack{p \mid m \\ p \leqslant D}} p
$$

We then associate with each integer $m$ an $(l-1)$-dimensional vector $\vec{\lambda}(m)$ as follows:

$$
\vec{\lambda}(m)=(\lambda(m+1), \lambda(m+2), \ldots, \lambda(m+l-1)) .
$$

Next, for a given vector $\vec{\lambda}=\left(\lambda_{1}, \ldots, \lambda_{l-1}\right)$, wherein each $\lambda_{i}$ is an integer which is a product of distinct primes $\leqslant D$, and

and

$$
\sum_{p \mid \lambda_{i}} \frac{1}{p}<E, \quad i=1, \ldots, l-1
$$

we estimate the number of $m \leqslant n$ satisfying (4.9) such that $\vec{\lambda}(m)=\vec{\lambda}$ (possibly none). For such $m$ we have

$$
m+i \equiv 0\left(\bmod \lambda_{i}\right),
$$$$
i=1, \ldots, l-1,
$$

so that if there are any solutions they belong to a single arithmetic progression modulo $[\vec{\lambda}]=\left\{\lambda_{1}, \ldots, \lambda_{l-1}\right\}$, the least common multiple of the $\lambda_{i}, i=1, \ldots, l-1$. Furthermore, in order that such solutions exist we must have

$$
\left(\lambda_{i}, \lambda_{j}\right) \mid i-j \quad i \neq j, 1 \leqslant i, j \leqslant l-1 .
$$

Suppose then that the aforementioned progression is $m \equiv \alpha(\bmod [\vec{\lambda}])$. For those $m$ such that $\vec{\lambda}(m)=\vec{\lambda}$ which satisfy (4.9) it follows that

$$
\frac{6}{\pi^{2}} l-2 \delta-\sum_{i=1}^{l-1} \frac{\phi_{D}\left(\lambda_{i}\right)}{\lambda_{i}} \leqslant \frac{\phi_{D}(m)}{m} \leqslant \frac{6}{\pi^{2}} l+2 \delta-\sum_{i=1}^{l-1} \frac{\phi_{D}\left(\lambda_{i}\right)}{\lambda_{i}},
$$


so that for these $m, \phi_{D}(m) / m$ lies in a fixed interval of length $4 \delta$ which we shall denote by $I_{\delta}=I_{\delta}(\vec{\lambda})$. Thus the number of $m \leqslant n$ such that $\vec{\lambda}(m)=\vec{\lambda}$ and which satisfy (4.9) equals the number of $m \leqslant n$ which satisfy
(a) $m \equiv \alpha(\bmod [\vec{\lambda}])$
(b) $\left(\frac{m+i}{\lambda_{i}}, \frac{\Delta}{\lambda_{i}}\right)=1$,
$i=1, \ldots, l-1 ; \Delta=\prod_{p \leqslant D} p$,
(c) $\frac{\phi_{D}(m)}{m} \in I_{\delta}$.

LEMma 4.2. Given any $\eta>0$, for $D$ fixed sufficiently large, and $\delta$ sufficiently small (these requirements are however independent of $\vec{\lambda}$ ), the number of $m \leqslant n$ such that (a), (b) and (c) hold is less than

$$
(\eta n /[\vec{\lambda}]) \prod_{p \leqslant D}\left(1-\frac{1}{p}\right)^{l-1} .
$$

Proof. Suppose that the above statement concerning the estimate (4.20) is false, so that for infinitely many $n$, the number of $m \leqslant n$ satisfying (a), (b) and (c) is more than

$$
\left(c_{3} n /[\vec{\lambda}]\right) \prod_{p \leqslant D}\left(1-\frac{1}{p}\right)^{l-1} .
$$

Let $z_{1}, z_{2}, \ldots$ be those integers, composed of primes $\leqslant D$, which can occur as divisors of an integer $m \equiv \alpha(\bmod [\vec{\lambda}])$ and such that (we denote the $z_{k}$ generically by $z$ )

$$
\frac{\phi_{D}(z)}{z}=\frac{\phi(z)}{z} \in I_{\delta}
$$

From (4.16), (4.19) and our assumption $\delta<\rho / 3$, (4.22) yields

$$
\frac{\phi(z)}{z} \geqslant \frac{\rho}{3} \text {. }
$$

Consider the number of $m \leqslant n$ such that (a), (b) above hold, and in addition for a fixed $z$,

$$
m \equiv 0(\bmod z),\left(\frac{m}{z}, \Delta\right)=1 .
$$

Clearly (d) implies (c).

Delete from $\Delta /[\vec{\lambda}]$ all prime factors $\leqslant l$ and any other prime factors of $z$; denoting the resulting integer by $\psi$. Then the number of $m \leqslant n$ which satisfy (a), (b), (d) is less than or equal to the number which satisfy (a) and

$$
(m+i, \psi)=1 \text {, }
$$$$
i=1, \ldots, l-1
$$

and

$$
m \equiv 0(z),\left(\frac{m}{z}, \psi\right)=1
$$


Setting $m=v z$ we have that the number of such $m$ equals

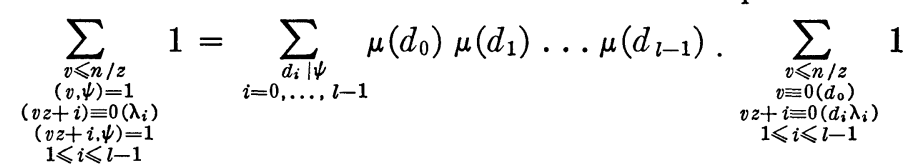

Since $\left(d_{i}, z\right)=1,\left(\lambda_{i}, \lambda_{j}\right) \mid i-j$, and the primes which divide $\psi$ are $>l$, we see that the system of congruences

$$
v \equiv 0\left(d_{0}\right), \quad v z+i \equiv 0\left(d_{i} \lambda_{i}\right), \quad 1 \leqslant i \leqslant l-1,
$$

has solutions if and only if

$$
\left(d_{i}, d_{j}\right)=1 \text {, and }\left(z, \lambda_{i}\right) \mid i ; \quad i \neq j, \quad 0 \leqslant i, \quad j \leqslant l-1 .
$$

Furthermore, if (4.25) holds we have, since $\left(d_{i}, \lambda_{j}\right)=1$,

Inserting this in (4.24) we get

$$
\begin{aligned}
\sum_{\substack{v \leqslant n / 2 \\
z=0\left(d_{0}\right) \\
z+i=0\left(d_{i}\right) \\
1 \leqslant i \leqslant l-1}} 1 & =\frac{n}{z} \frac{1}{\left\{d_{0}, \frac{d_{1} \lambda_{1}}{\left(z, \lambda_{1}\right)}, \ldots, \frac{d_{l-1}, \lambda_{l-1}}{\left(z, \lambda_{l-1}\right)}\right\}}+O(1) \\
& =\frac{n}{z d_{0} d_{1} \ldots d_{l-1}} \frac{1}{\left\{\frac{\lambda_{1}}{\left(z, \lambda_{1}\right)}, \ldots, \frac{\lambda_{l-1}}{\left(z, \lambda_{l-1}\right)}\right\}}+O(1) .
\end{aligned}
$$

Since

$$
M=\frac{n}{z} \cdot \frac{1}{\left\{\ldots, \frac{\lambda_{i}}{\left(z, \lambda_{i}\right)}, \ldots\right\}} \sum_{\substack{d_{i} i \psi \\\left(d_{i}, d_{i}\right)=1 \\ 0 \leqslant i, j \leqslant l-1}} \frac{\mu\left(d_{0}\right) \ldots \mu\left(d_{l-1}\right)}{d_{0} \ldots d_{l-1}}+O(1) .
$$

and from (4.15), (4.23)

$$
\begin{aligned}
& \sum_{\substack{d_{i} \mid \psi \\
\text { and } \\
0 \leqslant i, d_{j}=1 \\
0 \leqslant i \leqslant l-1}} \frac{\mu\left(d_{0}\right) \ldots \mu\left(d_{l-1}\right)}{d_{0} \ldots d_{l-1}}=\sum_{c \mid \psi} \frac{\mu(c)}{c} l^{\nu(c)}=\prod_{p \mid \psi}\left(1-\frac{l}{p}\right) \\
& <c_{4} \prod_{p \mid \psi}\left(1-\frac{1}{p}\right)^{l} \\
& <c_{5}\left(\frac{z}{\phi(z)}\right)^{l} \prod_{p \mid \vec{\lambda}]}\left(1+\frac{1}{p}\right)^{l} \prod_{p \leqslant D}\left(1-\frac{1}{p}\right)^{l}
\end{aligned}
$$

$$
\begin{aligned}
& \prod_{p \mid \overrightarrow{\mid \vec{\lambda}}}\left(1+\frac{1}{p}\right)^{l} \leqslant e^{E l^{2}}, \\
& \left(\frac{z}{\phi(z)}\right)^{l} \leqslant\left(\frac{3}{\rho}\right)^{l},
\end{aligned}
$$

(4.26) yields (since $\left(z, \lambda_{i}\right) \mid i$ )

or

$$
M<c_{6}\left(\frac{3}{\rho}\right)^{l} e^{E l^{2}} l !(n / z[\vec{\lambda}]) \prod_{p \leqslant D}\left(1-\frac{1}{p}\right)^{l}
$$

$$
M<c_{7}(n / z[\vec{\lambda}]) \prod_{p \leqslant D}\left(1-\frac{1}{p}\right)^{l}
$$

where $c_{7}>0$ is independent of $D$. 
(4.27) together with (4.21) implies

$$
\sum_{k} \frac{1}{z_{k}} \prod_{p \leqslant D}\left(1-\frac{1}{p}\right)>\frac{c_{3}}{c_{7}} .
$$

On the other hand, the number of $m \leqslant n$ such that (d) holds for some $z_{k}$ is, for large $n$, greater than or equal to

$$
\frac{n}{2} \sum_{k} \frac{1}{z_{k}} \prod_{p \leqslant D}\left(1-\frac{1}{p}\right)>\frac{c_{3}}{2 c_{7}} n,
$$

using (4.28). Since for these $m, \phi_{D}(m) / m$ lies in a fixed interval $I_{\delta}$ of length $4 \delta$, we see that for at least $c_{3} n / 4 c_{7}$ of these $m, \phi(m) / m$ lies in a fixed interval of length $8 \delta$ (if $D$ is large enough). Since $c_{3} / 4 c_{7}$ is independent of $\delta$ (and of $D$ ), this would contradict the continuity of the distribution function of $\phi(m) / m$. Thus the lemma is proved.

Finally then, letting $T$ denote the number of $m \leqslant n$ which satisfy (4.9), (4.11) and (4.14), we have

Since

$$
T<\eta n \prod_{p \leqslant D}\left(1-\frac{1}{p}\right)^{l-1} \sum_{\vec{\lambda}}[\vec{\lambda}]^{-1} .
$$

we have

$$
\begin{aligned}
\sum_{\vec{\lambda}}[\vec{\lambda}]^{-1} & \leqslant c_{8}\left(\sum_{\lambda \mid \Delta} \frac{1}{\lambda}\right)^{l-1} \\
& \leqslant c_{8} \prod_{p \leqslant D}\left(1+\frac{1}{p}\right)^{l-1},
\end{aligned}
$$

$$
T<c_{9} \eta n \text {. }
$$

But for $\eta$ sufficiently small, $c_{9} \eta<A^{2} / 128$, so that we obtain a contradiction, and the proof is completed.

\section{REFERENCES}

1. A. Walfisz, On Euler's functions, Akad. Nauk USSR, 90.4 (1953), 491-493.

2. - Teilerprobleme II, Math. Z., 34 (1931), 448-472.

3. S. S. Pillai and S. D. Chowla, On the error term in some asymptotic formulae in the theory of numbers I, J. London Math. Soc., 5 (1930), 95-101.

4. P. Erdös, H. N. Shapiro, On the changes of sign of a certain error function, Can. J. Math., 8 (1951), 375-383.

5. P. Erdös, On the density of some sequences of numbers III, J. London Math. Soc., 13 (1938), 119-127.

6. P. Erdös and A. Wintner, Additive arithmetical functions and statistical independence, Amer. J. Math., 61 (1939), 713-721.

7. S. Chowla and P. Erdös, $A$ theorem on the distribution of the values of L-functions, J. Indian Math. Soc., 15 (1951), 11-18.

8. S. Chowla, Contributions to the analytic theory of numbers, Math. Z., 85 (1932), 280-299.

Notre Dame University

New York University 